\title{
Implementation and Performance of Fast Parallel Multi-Baseline Stereo Vision
}

\author{
Jon A. Webb \\ School of Computer Science \\ Carnegie Mellon University \\ Pittsburgh, PA 15213-3890
}

\begin{abstract}
A fast implementation of multi-baseline stereo vision on a parallel computer is described. For three $240 \times 256$ images, the algorithm runs in $64 \mathrm{~ms}$ on 64 iWarp processors, exceeding $15 \mathrm{~Hz}$ frame rate. This is a speedup of 51 over an implementation on a SPARC II and represents the fastest correlation-based stereo vision system reported. Implementing this algorithm this efficiently required careful trade-offs in algorithm design and, particularly, in the implementation of the basic communication operations. A building block approach is described for achieving best efficiency in communication; the basic operations that the parallel computer can do at maximum speed are identified, and then these primitives are used to construct the communications functions needed by the algorithm.
\end{abstract}

\section{Introduction}

In robotics applications processing goes through a sense-plan-act loop as illustrated in Figure 1.

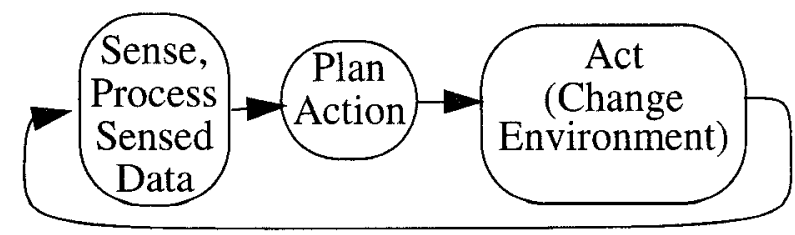

Figure 1. Sense-plan-act cycle in robotics applications.

We would like to speed up this cycle through the use of parallel computers. A major bottleneck in this cycle is processing of sensory data, particularly when the sensor is a

This research was supported by the National Science Foundation under Grant MIPS 8920420 and by the Defense Advanced Research Projects Agency and monitored by U. S. Army Training and Doctrine in Fort Huachuca, Arizona under Contract DABT63-91-C-0035.

The Government has certain rights in this material. Any views, opinions, findings, and conclusions or recommendations expressed in this material are those of the author and do not necessarily reflect the views of the National Science Foundation. They should also not be interpreted as representing the official policies, either expressed or implied, of the U.S. Government. camera, since images are so large. This paper discusses a fast parallel implementation of stereo vision.

The robot must sense the results of its actions after they take place. The sensory processing must take place before action planning. Hence it is the total execution time of sensory processing that is important (its latency), not the number of sensory actions completed per second (its throughput).

A major bottleneck in parallel processing is message passing latency and bandwidth. As we apply more processors to a problem, message passing latency and bandwidth stay fixed for a given architecture, and, since more processors imply more communication, come to dominate total processing

We have achieved the highest reported performance ever of a stereo vision system based on correlation: $64 \mathrm{~ms}$ for $240 \times 256$ imagery, exceeding. $15 \mathrm{~Hz}$ on a 64-processor iWarp computer. Achieving this was not simply a matter of increasing processor performance but involved many careful trade-offs that apply to vision systems and parallel machines in general. This paper closely analyzes the performance of this one system in order to derive general lessons concerning the performance of parallel systems on computer vision problems.

\section{Stereo vision algorithm}

The stereo vision algorithm used here is Kanade-Okutomi multi-baseline stereo [2]. Multi-baseline stereo uses multiple cameras aligned so that all that differs between the cameras is the baseline separation between them. This provides redundant information in a way that is very easy to exploit, allowing a simple matching algorithm to give very good results.

The principle of multi-baseline stereo is illustrated in Figure 2. In ordinary stereo, there are two images, a reference and a match image. For each pixel in the reference image, and for each possible disparity, the error is calculated between the reference and match images.

Ordinary stereo can give multiple false matches, as illustrated. Multi-baseline stereo reduces this problem by 


\section{Ordinary Stereo}
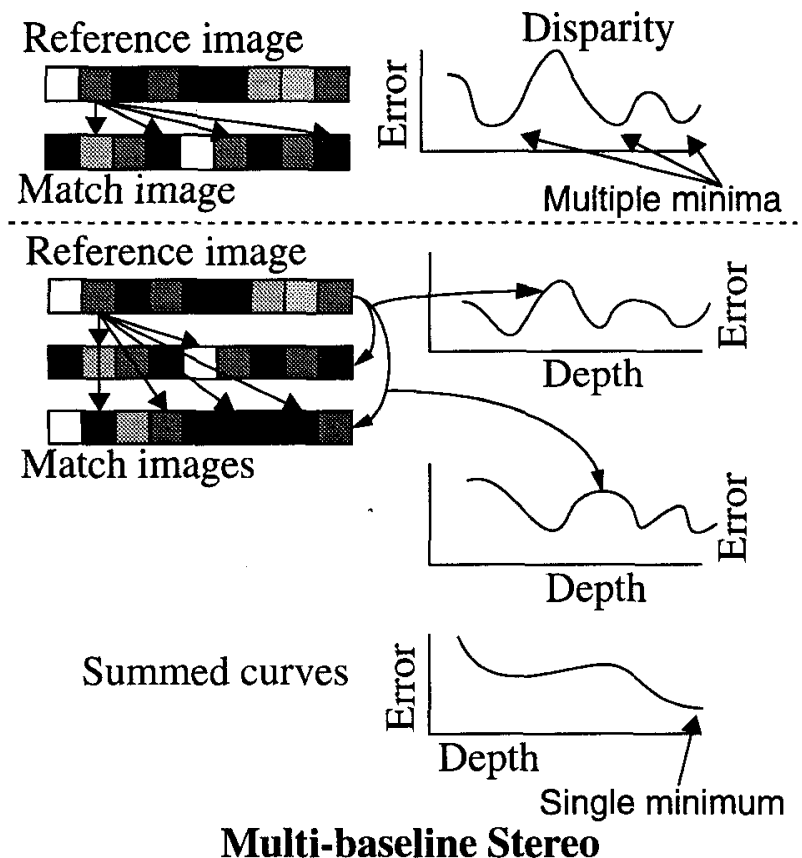

Figure 2. Ordinary and multi-baseline stereo.

taking advantage of redundant information. The principle is that if we graph the error function in the match between any two cameras according to depth, not disparity, then a false match has no better than chance likelihood of falling at the same depth for any two pairs of cameras. But the true match will fall at the same depth for all camera pairs. Therefore, by simply adding the error functions for all camera pairs, false matches will cancel each other out and the only minimum will appear at the true match.

The error is calculated by summing the error between the images over a small window of size $h \times w$. With a naive algorithm, this calculation over a image of size $r \times c$ will take $r c h w$ add operations. By maintaining row and column sums, we can reduce this to $4 r c$ operations plus some overhead. However this also reduces the available parallelism, because the row and column sums must be updated sequentially.

\section{Parallel design issues}

Adapt was used to implement the stereo vision algorithm. Adapt is a specialized language for image processing on parallel computers that has been extensively described elsewhere [3]. The Adapt compiler automatically parallelizes image processing programs, but requires the programmer to write them in terms of a series of image to image operations, each of which is described as a program to be applied at every pixel or every row of the images in parallel.

Multi-baseline stereo vision divides naturally into two steps, which are repeated for each disparity $d$ :

1. Difference. Calculate the difference image, which is the pixel-by-pixel error between the reference image and the match images. This is done by forming the sum of squared differences between the reference pixel and the match images shifted within rows by appropriate multiples of $d$.

2. Minimize. At each pixel, sum the difference image over the $h \times w$ window and compare this with the best error so far. If less, replace the error value by the new value and the previous disparity by $d$.

The difference step can be implemented easily and efficiently in Adapt. However, if the minimize step is implemented in the obvious way processor utilization is poor. This is because the number of rows $h$ in the window is generally large compared to the number of rows per processor; for example, the image size may be $240 \times 256$ and there are 64 processors, while the window size is $13 \times 13$. When the window sum is formed, each processor will be repeating the work of other processors with overlapping rows. As a result, there will be little or no speedup as the number of processors is increased beyond the point at which the number of rows per processor equals the window height, or about 18 processors in this case.

The solution to this is to pre-calculate the partial sum of the difference image in the difference step. Each processor can simply add its four rows together, forming a sum image, which is distributed to other processors before the minimize step. The minimize step then forms its initial row sum by adding in appropriate rows of the sum image, together with whatever image rows are needed to make the window height exactly $h$ rows.

In order to specify the algorithm further, the Adapt code for difference and minimize is given in Appendix I. This code omits the partial summation described in the last paragraph as it complicates understanding.

\section{Computation Issues}

Table 1 summarizes the performance of the computation in the stereo program and compares the performance of the assembly-language routines used with $\mathrm{C}$-generated code (both the assembly and $\mathrm{C}$ routines are called from a modified version of the Adapt code in Appendix I).

\begin{tabular}{|c|c|c|}
\hline Language & Assembly & $C$ \\
\hline Difference & $22.0 \mathrm{~ms}$ & $36.0 \mathrm{~ms}$ \\
& $(257 \mathrm{MFLOPS})$ & $(157 \mathrm{MFLOPS})$ \\
\hline Minimize & $17.3 \mathrm{~ms}$ & $40.7 \mathrm{~ms}$ \\
& (369 MFLOPS) & (156 MFLOPS) \\
\hline Total & $39.3 \mathrm{~ms}$ & $76.7 \mathrm{~ms}$ \\
\hline
\end{tabular}

Table 1. Stereo Computation Times for three $240 \times 256$ images and 16 disparity levels, on 64 processors. 
There are two reasons why the assembly code is significantly faster than the C code: (1) The compiler optimizes code primarily within a basic block. In assembly coding, there is no such restriction. (2) Bugs in the C-step implementation of the iWarp component, impact the performance of the compute-and-access instruction, which allows floating point add and multiply operations to proceed in parallel with memory access operations. Since the $\mathrm{C}$ compiler must generate code for the most general case, it cannot generate the most efficient code. However, within Adapt these bugs either cannot occur or can easily be avoided.

\section{5. iWarp Communications Structure}

Before discussing communications issues in the stereo program, we first discuss the communication capabilities of the iWarp computer; more detail is available elsewhere [1]. It is a two-dimensional torus, each of which is connected to its nearest neighbor by input and output ports, each of which can transfer data at $40 \mathrm{MB} / \mathrm{s}$. Cell $(0,0)$ of the processor array is connected to the SIB, a special iWarp processor that can communicate with I/O devices across a VME bus; all data processed by the iWarp array must pass through the SIB.

iWarp has a three-level communications structure, as illustrated in Figure 4. It supports connections which define communications routes among processors. Connections

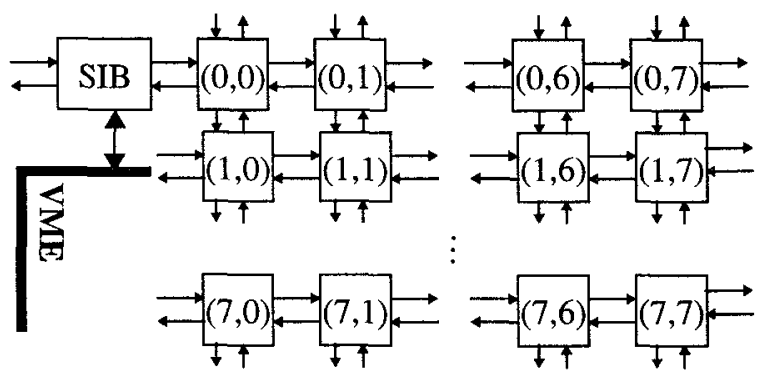

Figure 3. iWarp Physical Layout

are set up at compile time. Within connections messages can be used to allow more than two processors to communicate on a single connection. Within messages, data can be sent word by word (called systolic) or with a DMA-like operation called spooling which transfers the data in the background.

\section{Adapt Implementation on iWarp}

In order to completely specify the parallel implementation of the stereo algorithm on iWarp, we will describe the Adapt implementation of any image processing program on this machine.

Adapt uses a mater-slave model. A master (sequential) program, written in $\mathrm{C}$, runs on the $\mathrm{SIB}$, coordinates input

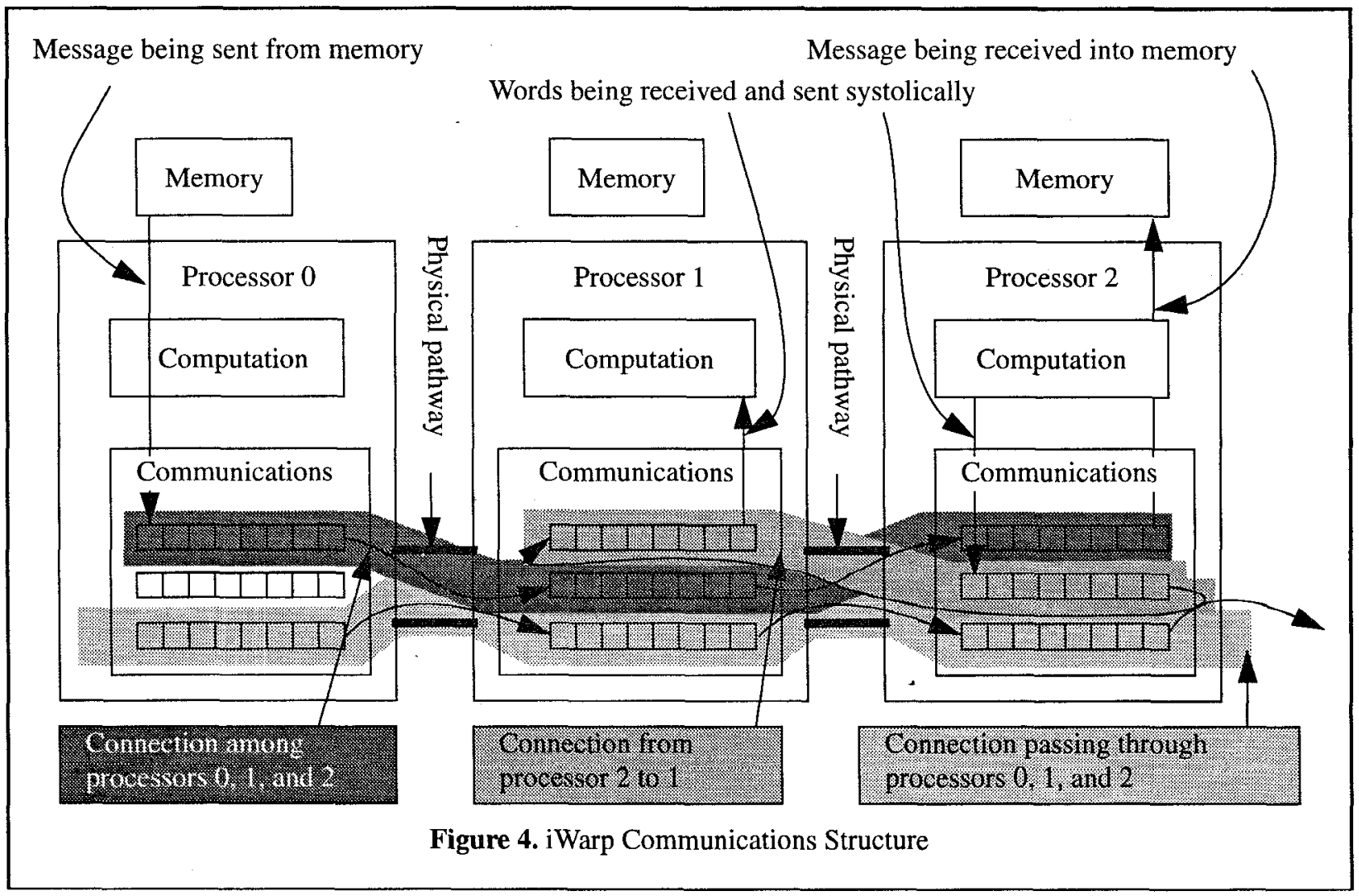


and output of images, and calls Adapt routines on cells $(0,0)$ through $(7,7)$ (the slave cells) to do all image processing. The Adapt routines consists of a stub $\mathrm{C}$ routine that runs on the SIB (which is called from the user program); this stub routine broadcasts the user parameters to the main body of the Adapt program, which is a $\mathrm{C}$ program running on the slaves. Both of these $\mathrm{C}$ programs are generated by the Adapt compiler from the user's program.

Images are distributed by rows on the processor array, by blocks; cell $(0,0)$ takes the first $1 / 64^{\text {th }}$ of the rows, cell $(0,1)$ the second $1 / 64^{\text {th }}$, etc. All Adapt-generated programs take as parameters images already distributed on the processor array; special Adapt I/O functions distribute images from the SIB to the other processors and collect images from the other processors to the SIB's memory.

One of the first things any Adapt-generated program running on the slaves has to do after receiving its parameters is to create a working copy of the input images. Commonly, a window of pixels is required for processing around a given pixel in an image processing program. The pixels in this window must be obtained by communication with other processors.

\section{Communications Issues}

The speed of Adapt programs depends on the speed of the underlying computation (which is the result of user choices and the $\mathrm{C}$ compiler, neither of which is under Adapt's control) and the Adapt I/O functions. Optimizing Adapt's I/O functions has been a subject of some considerable effort. It was not, however, until the building block approach, to be described in this section, was tried that truly efficient implementations were found, and we were confident that these implementations were near the best possible.

As described in the last section, there are four Adapt important $\mathrm{I} / \mathrm{O}$ functions:

1. Broadcast. Send parameters (generally a small amount of data) from the master to the slaves.

2. Distribute. Send an image from the master to the slaves. Each slave gets one block of rows of the image.

3. Collect. The inverse of distribute; return an image distributed by rows on the slaves to the master.

4. Create working copy. Exchange rows between the slaves so that each has the rows needed to fill the window specified for that image in the Adapt program.

Each iWarp cell has eight physical pathways, each with a bandwidth of $40 \mathrm{MB} / \mathrm{s}$. (On the SIB, only four of these pathways are in use, connected to cells $(0,0)$ and $(0,7))$. The maximum physical bandwidths for these functions are therefore:
- Broadcast: $40 \mathrm{MB} / \mathrm{s}$. (While there are two pathways from the SIB to any cell, the maximum bandwidth on any pathway is $40 \mathrm{MB} / \mathrm{s}$, so this is the limit. We cannot send data on both pathways because this would mean breaking a small amount of data (the parameter block) into two parts and sending the two halves by two different routes to every cell, which would lead to excessive overhead).

- Distribute: $80 \mathrm{MB} / \mathrm{s}$. (Each cell receives different data from every other cell. It is possible to use both pathways from the SIB by dividing the cells into two groups, one which receives data by two different routes).

- Collect: also $80 \mathrm{MB} / \mathrm{s}$

- Create working copy: $160 \mathrm{MB} / \mathrm{s}$ (by using all four physical pathways connecting adjacent cells).

These bandwidths are achieved (to within about 10\%) in our implementation of Adapt on iWarp, even for relatively small amounts of data. To do this, we define primitive maximum bandwidth operations (that is, I/O operations which the iWarp processor can execute at maximum bandwidth) and then implement all communications operations in terms of them, ensuring maximum performance for communications. The primitive $\mathrm{I} / \mathrm{O}$ operations for iWarp are:

1. Receive. Data is taken from the pathway and stored in memory.

2. Send. Data is taken from memory and sent to the pathway,

3. Pass. Data is passed from one pathway to another.

4. Receive and pass. Data is be passed from one pathway to another and simultaneously copied into memory.

5. Receive and pass twice. Data is be copied into memory and also sent to two other pathways.

\subsection{Broadcast}

Broadcast can be implemented in a variety of ways. The SIB is connected to the processor array at two points, so data can be sent either entirely from one connection or over both connections simultaneously. Once the data is inside array it can be transferred from cell to cell in a pipeline using receive and pass or in a tree fashion by also using receive and pass twice. We will consider these three techniques: using one SIB connection and pipelined transfer within the array (method (a)); using both SIB connections and pipelined transfer (method (b)); using one SIB connection and tree transfer within the array (method $(\mathrm{c}))$.

All of these methods achieve the same maximum bandwidth. The only difference between them is in latency. These differences in latency lead to a significant difference in performance, as illustrated in Figure 5. 


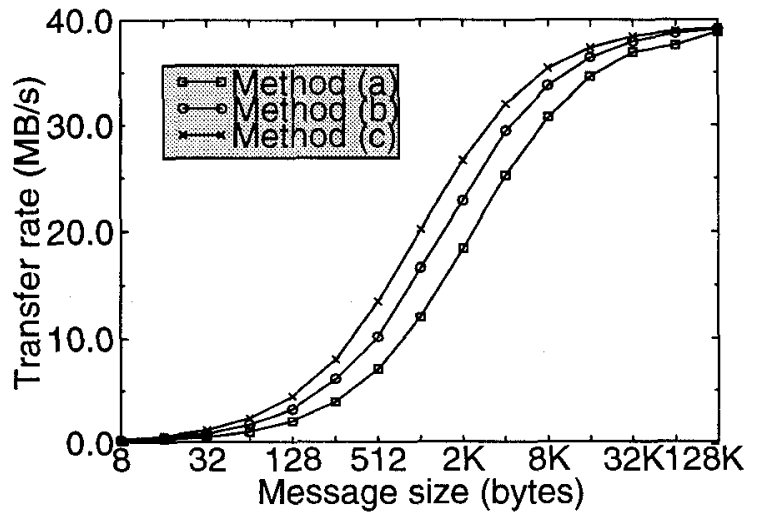

Figure 5. Performance of different implementations of broadcast.

Adapt uses method (c) for broadcast.

\subsection{Distribute}

There are only two fundamentally different methods of implementing distribute. In the first method, data is sent systolically from the SIB over one pathway; each cell executes a receive followed by a pass. In the second method data is sent from the SIB over two pathways, one that goes in a forward direction through the processors and the other that goes in a reverse direction. Processors in the forward pathway act as before, while processors in the reverse pathway execute at pass followed by a receive.

Since the second method has a maximum physical bandwidth of $80 \mathrm{MB} / \mathrm{s}$ versus $40 \mathrm{MB} / \mathrm{s}$ for the first method, it is to be preferred if it does not impose too high startup overhead. We therefore examine the transfer rate for images of various sizes in Figure 6. From this graph we observe that even for small images of $8 \mathrm{~K}$ bytes, the transfer rate exceeds the maximum that can be expected from using just one pathway. We therefore adopt the two pathway method.r

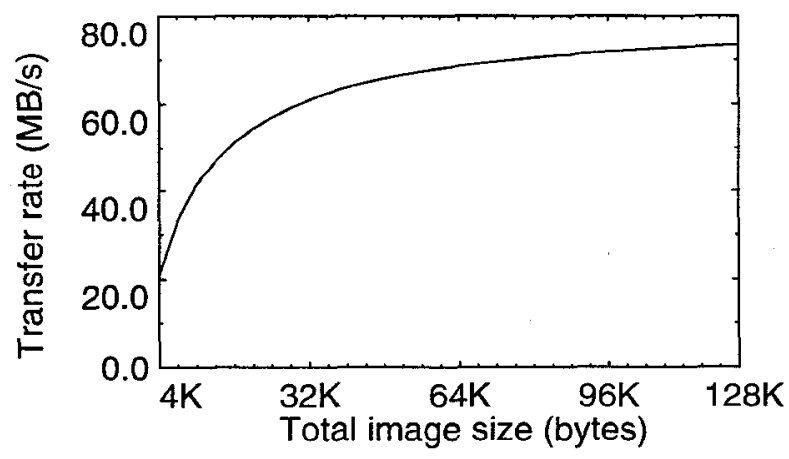

Figure 6. Performance of distribute.

\subsection{Collect}

Collect can be implemented using one or two pathways, just as with distribute. The performance of the two pathway method is shown in Figure 7. As with distribute, the graph rapidly exceeds the maximum bandwidth with a single pathway (at $12 \mathrm{~K}$ bytes the bandwidth is $42 \mathrm{MB} / \mathrm{s}$ ), so this method is chosen. However, the maximum bandwidth achievable in collect seems to be $60 \mathrm{MB} / \mathrm{s}$, which is less than the theoretical maximum of $80 \mathrm{MB} / \mathrm{s}$. This is due to a bug in the iWarp hardware that limits the maximum physical bandwidth while transferring into the SIB to $30 \mathrm{MB} / \mathrm{s}$ over any one physical pathway.

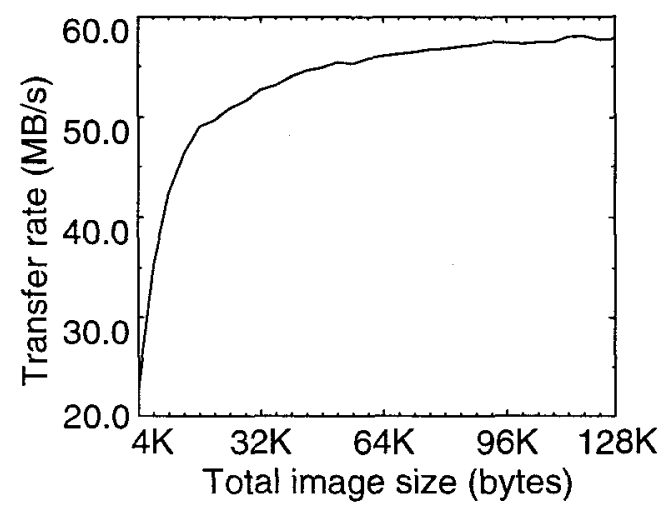

Figure 7. Performance of collect.

\subsection{Create working copy}

In create working copy each cell initially has several rows of data, and will obtain some rows of data above and below its rows from adjacent cells. The behavior is shown in Figure 8. From this figure, it is clear that create working copy can be implemented as two shift operations, in which processor $i$ sends its rows of data to the next or previous processor in the array, depending on whether the shift is in a forward or reverse direction. Create working copy consists of one shift in the forward direction and one shift in reverse.

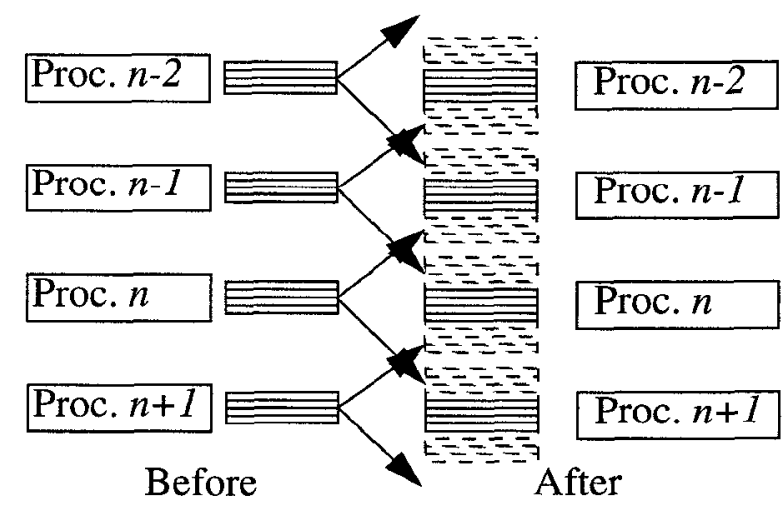

Figure 8. Create working copy. 
Each shift operation can be implemented with send and receive operations in the obvious way. Using spooling, each processor can be sending and receiving data for both shifts simultaneously, giving a maximum bandwidth of 160 $\mathrm{MB} / \mathrm{s}$.

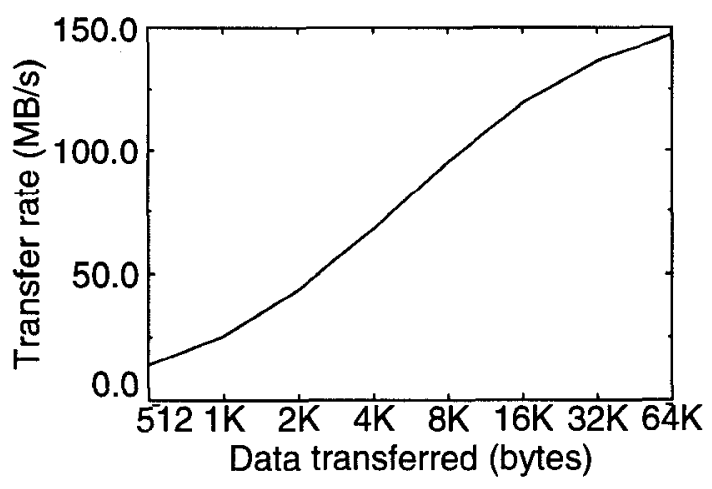

Figure 9. Performance of create working copy.

The performance of the I/O in create working copy is shown in Figure 9. The transfer rate seems to peak at 150 $\mathrm{MB} / \mathrm{s}$, instead of the expected $160 \mathrm{MB} / \mathrm{s}$; some of this difference must be due to the same hardware limitation that led to the maximum $60 \mathrm{MB} / \mathrm{s}$ transfer rate to the SIB in collect.

\subsection{Stereo I/O times}

Table 2 summarizes the stereo I/O times. The times for distribute and create working copy were measured directly, and the times for broadcast and collect were estimated from the work earlier in this section (these times could not be estimated directly in a running system since they are overlapped with other computation; thus, the times reported here are overestimates in terms of the impact of these times on total execution time.)

\begin{tabular}{|c|c|c|}
\hline \multicolumn{2}{|c|}{ Broadcast } & $1.92 \mathrm{~ms}$ \\
\hline \multicolumn{2}{|c|}{ Distribute } & $3.00 \mathrm{~ms}$ \\
\hline $\begin{array}{c}\text { Create } \\
\text { working copy }\end{array}$ & difference image & $8.76 \mathrm{~ms}$ \\
\cline { 2 - 3 } & sum image & $4.90 \mathrm{~ms}$ \\
\hline \multicolumn{2}{|c|}{ Collect } & $4.16 \mathrm{~ms}$ \\
\hline \multicolumn{2}{|c|}{ Total } & $22.7 \mathrm{~ms}$ \\
\hline \multicolumn{2}{|c|}{} \\
\hline
\end{tabular}

Table 2. Stereo I/O times for three $240 \times 256$ images and 16 disparity levels

\section{Scaling with number of processors}

Figure 10 gives the scaling of the stereo vision algorithm (for three $240 \times 256$ images with a $13 \times 13$ summation window) from 16 to 64 processors. The execution time does not decrease smoothly with increasing numbers of processors because the program is sensitive to the match between the number of rows in the image and the number of processors. In the pre-calculation of the sums of the difference image described at the end of Section 3, if the number of processors does not nearly evenly divide the number of image rows divided by four (i.e., 60) then the image is automatically shifted within the processor array. This is an expensive operation (in this case, the image is shifted to cells $0-29$, numbered in raster order) that leads to a significant loss of efficiency with processor numbers of 40,48 , and 56.

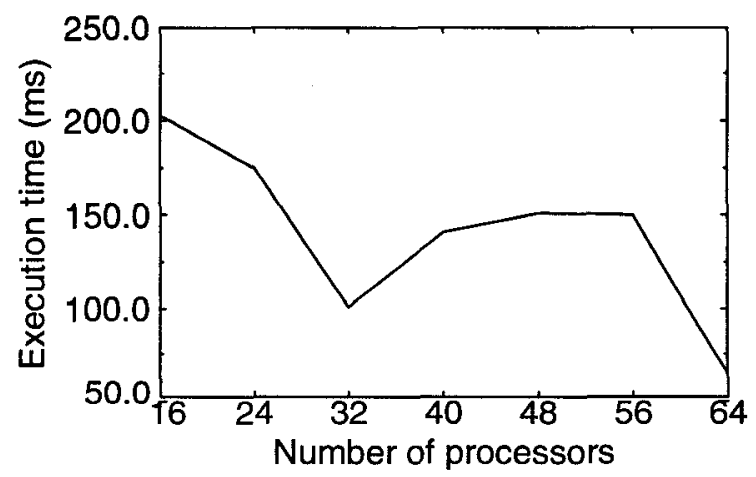

Figure 10. Scaling of performance with number of processors.

\section{Video Interfaces}

The algorithm described in this paper runs at $15 \mathrm{~Hz}$; but in order to demonstrate this performance, we must connect the iWarp system to cameras with low enough latency and high enough bandwidth. This is a difficult issue in itself. We have three different systems that we think could be used to demonstrate real-time performance. They are illustrated in Figure 11.

In the first system, which uses only commercial hardware, an Ironics IV-FCFG framegrabber is used for image capture and display. We have measured a transfer rate of 5.12 MB/s between the Ironics and the iWarp SIB over the VME bus. At this transfer rate, we can achieve a processing speed of 8-10 Hz depending on whether we can display the processed image in a buffer of the Ironics while capturing the next image in a different buffer in parallel, or must display the processed image in a buffer after waiting for the input image to be captured.

In the second and third systems we achieve the full processing rate by dividing the image capture and display between two boards, and by avoiding using the VME bus for transferring both images. This allows both operations to go on in parallel. In the second system we use a custombuilt iWarp memory interface, which allows the direct $A / D$ conversion of data from the camera and distribution to other iWarp cells under control of the iWarp processor (the board we use actually has four camera input ports rather than the three illustrated, and receives the video input as four bytes packed into a 32-bit word). We also employ a 


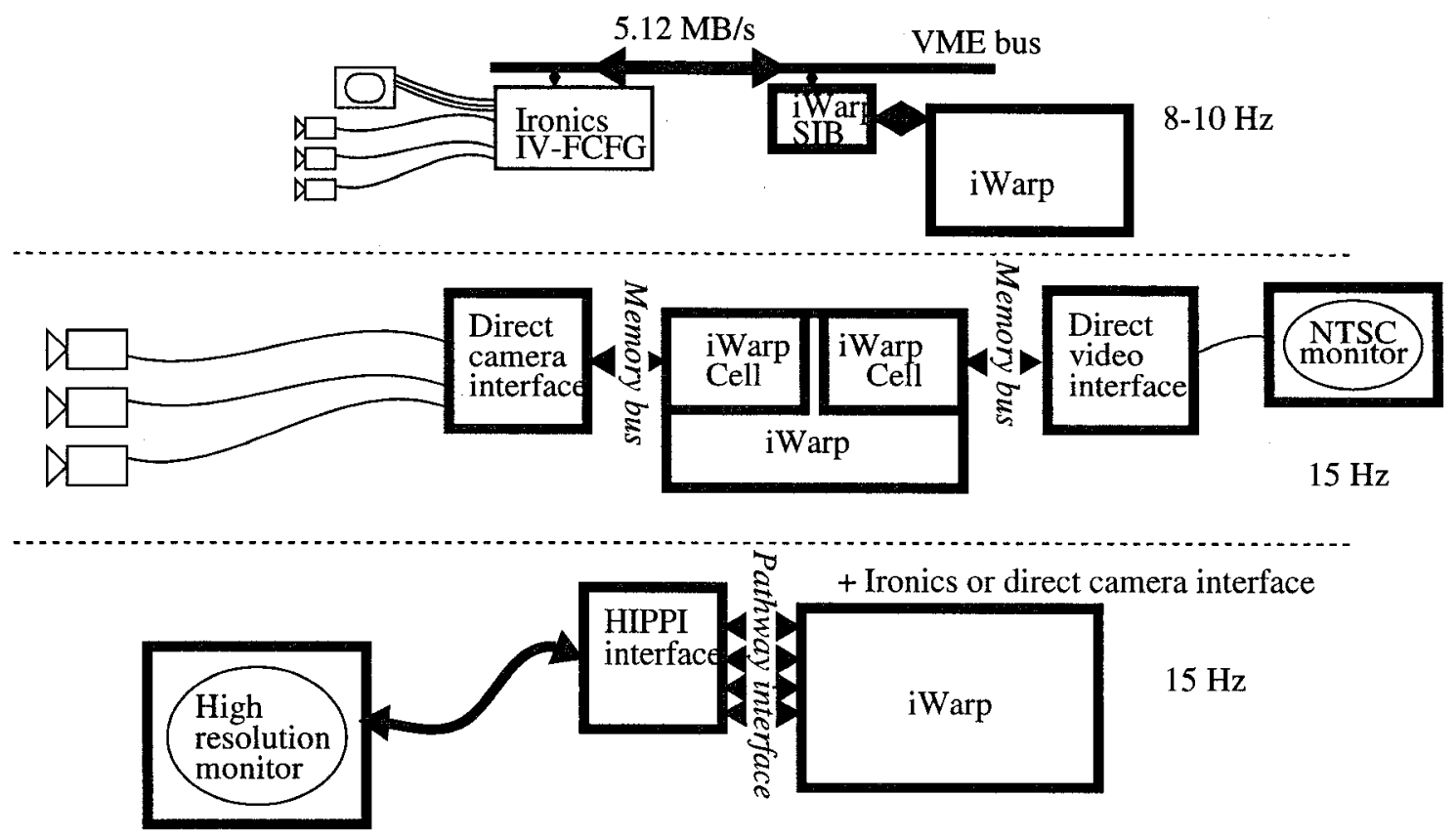

Figure 11. Interfaces to fast stereo vision.

second custom-built board that performs D/A conversion and output to an NTSC monitor. These boards were very simple to design and build because they use the iWarp processor to do all video control; at $20 \mathrm{Mhz}$, the iWarp processor is just fast enough to keep up with the demands of driving a display or receiving data from cameras. Either board continually receives or sends images to the external device, transferring the data sent or received to a second iWarp processor cell adjacent to it in the array, using systolic I/O. This second cell, which acts as a framebuffer, transfers its data to or from the SIB as the SIB requests it. The SIB transfers data to the slave cells as described in Sections 6 and 7 . Note that the presence of these special function I/O cells in the middle of the iWarp array means that the $\mathrm{I} / \mathrm{O}$ pathways for Adapt must be routed around these cells; this is not a problem on iWarp because the virtual pathways can be set up in advance.

We could also have (and did, in an earlier implementation) achieved a $15 \mathrm{~Hz}$ processing rate by combining the use of the Ironics framebuffer with either the direct camera or the direct video interface board.

In the third system we use a HIPPI (high performance parallel interface) board built as part of the Nectar project by Network Systems Corporation, together with either of the options for video input. This interfaces to the iWarp pathway (by way of an auxiliary iWarp cell) and has achieved a transfer rate of $75 \mathrm{MB} / \mathrm{s}$ (sustained). Using a
HIPPI framebuffer, we should be able to display images at full rate while transferring them from the Ironics using the SIB. This is an expensive solution, since HIPPI framebuffers are expensive, and not as scalable as the second system (which can be expanded simply by adding more video output boards to other iWarp cells). But the HIPPI frame buffer is higher quality (higher resolution and color, rather than NTSC black and white, which is all we can achieve with the direct video board).

Using the direct camera interface and video input boards, we have achieved $15 \mathrm{~Hz}$ processing rate for $240 \times 256$ images and 16 disparity levels. We also achieved this processing rate using the direct camera interface and the Ironics for display.

\section{Conclusions and Future Work}

The stereo vision system described here is the fastest system for obtaining depth data presently available. It is even faster than laser ranging systems, for a comparable number of depth measurements. Achieving this high performance was not simply a matter of reimplementing an existing algorithm on a parallel computer, but rather required rethinking of the algorithm in one step, and particular attention to $\mathrm{I} / \mathrm{O}$ within the algorithm:

- The introduction of the sum image in the difference step made it possible to scale the algorithm beyond 
18 processors.

- Thinking of the I/O in iWarp in terms of a collection of primitive operators that could be assembled to get the Adapt I/O functions was the key to efficiency. Previous implementations of the Adapt I/O functions made apparently reasonable choices (e.g., using a binary tree and message-passing to implement broadcast) but because there was no systematic.approach to getting maximum efficiency performance was poor, and, more importantly, it was impossible to determine whether the implementation was as good as possible. In the work reported here we are reasonably certain that the Adapt $1 / O$ functions are as efficient as possible.

- Most parallel computers support only message passing, and have no systolic communication facilities. This limits the primitive operators to receive and send. Using just these operators, it is possible to build the Adapt I/O functions, but with great loss of efficiency, especially in broadcast. Perhaps by analysis of the primitive $\mathrm{I} / \mathrm{O}$ functions the machine is capable of as it is being constructed, increased flexibility can be introduced, resulting in greater efficiency for common program communications functions.

Our current research focuses on extending this work to build a high speed, high resolution stereo imaging system. Our goal is to obtain $1 \mathrm{~mm}$ accuracy in a $0.5 \mathrm{~m}$ cube - in other words, a $512 \times 512 \times 512$ depth image. We believe it should be possible to do this, using an improved camera configuration, manipulation of the environment (i.e., projecting a grid onto the scene), improvements in the stereo algorithm, and more powerful computing.

\section{Acknowledgments}

The work on stereo vision was started by Hans Thomas and Bill Ross of the Vision and Autonomous Systems Center at Carnegie Mellon. The Adapt I/O library was originally written by Doug Smith of the Adapt project. Michael Mills of the iWarp project did some of the early work on the iWarp implementation of multi-baseline stereo. The direct camera and video interface boards were built by Luke Tuttle and Thomas Warfel of the iWarp project. The support of several other members of the iWarp group, in particular Thomas Gross, Dave O'Hallaron, and Susan Hinrichs, is gratefully acknowledged. All graphs in this paper were prepared using ACE/gr, a public domain freeware tool written by Paul J. Turner of the Oregon Graduate Institute.

\section{Bibliography}

[1] Borkar, S., et al. Supporting Systolic and Memory Communication in iWarp. Proceedings of the 17th Annual International Symposium on Computer Architecture. Seattle, Washington, May, 1990.
Okutomi, M., and Kanade, T. A Multiple Baseline Stereo. Proceedings of the 1991 IEEE Computer Society Conference on Computer Vision and Pattern Recognition, pages 63-69. IEEE Computer Society, Lahaina, Maui, Hawaii, June, 1991.

[3] Webb, J. A. Steps Toward Architecture Independent Image Processing. IEEE Computer. 25(2):21-31, February, 1992. 


\section{Appendix I. Adapt Code for difference and minimize}

-- This code is designed for a camera

-- setup where the cameras are placed

-- at positions $0, x$, and $2 x$. With

-- this setup, all calculations can

- be done in terms of integral

- disparities (not depths) and no

-- sub-pixel interpolation is necessary.

- This code also does not include

- the summing of four rows of the

- difference image into one that is

-- described in Section 3.

procedure differencel

reference, match1, match2 :

in image array (0..o, width-1)

of byte sample(1, Width),

diff :

out image array $(0 \ldots 0,0 \ldots$ width-1)

of float,

is

disparity : in integer)

next begin

for $i$ in 0 .. Width-disparity*2 loop $\operatorname{diff}(0, i):=$ (reference $(0, i)$ $\operatorname{match} 1(0$, disparity $+i)) \star \star 2$

+ (reference $(0, i)$ $\operatorname{match} 2(0,2 * \operatorname{disparity+i)}) * * 2$;

end loop;

end next;

end difference;

procedure minimizel

diff :

in image

array (HalfVWin. .HalfVWin+1, 0 . Wiath-1)

of float sample(1, Width),

old_disp :

in image array $(0 \ldots 0,0 \ldots$ Width-1)

of integer sample(1,Width),

olderror :

in image array $(0 \ldots 0,0 \ldots$ Width-1)

of float sample(1, width),

new_disp :

out image array $(0 \ldots 0,0 \ldots$ Width-1)

of integer,

new_error :

out image array $(0 \ldots 0,0$. Width-1)

of float,

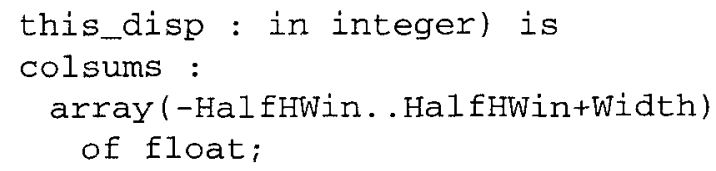

end loop;

for $i$ in 0 .

Width-HalfHWin-this_disp*2-2 loop

if this_error $<\operatorname{error}(0, i)$ then new_error $(0, i):=$ this_error; new_disp $(0, i):=$ this_disp;

else

new_error $(0, i):=01 d$ error $(0, i)$; new_disp $(0, i):=$ old_disp $(0, i)$; end if;

this_error := this_error colsums ( $i-H a l f H W i n)+$ colsums ( $i+$ Hal fHWin+1);

end loop:

for $i$ in 0. Width-this_disp*2 loop colsums $(i):=\operatorname{colsums}(i)$ diff (-HalfVWin, i) + diff (HalfVWin+1, i);

end loop;

end next;

end minimize; 